\title{
Dynamic Nuclear Polarization with Solid-State Millimeter Waves, 3D Printed Components, and Software-Defined-Radio-based NMR
}

\author{
Elena Long* and Karl Slifer \\ University of New Hampshire \\ E-mail: elena.long@unh.edu, karl.slifer@unh.edu
}

\begin{abstract}
A new dynamic nuclear polarization (DNP) target system has recently come on-line at the University of New Hampshire. DNP is driven by a novel solid-state $140 \mathrm{GHz}$ mm-wave source with quasi-optics transmission and low-loss $(<0.1 \mathrm{~dB} / \mathrm{m})$ overmodal waveguide that is insensitive to magnetic fields. We have also developed a method to 3D print with Kel-F, which was used to produce target cups utilized in a recent cool-down. Polarization measurements are made and cross-compared on a Liverpool Q-meter, LANL Q-meter, and low-cost software-defined-radio based vector network analyzer.
\end{abstract}

The 18th International Workshop on Polarized Sources, Targets, and Polarimetry, PSTP2019

23-27 September, 2019

Knoxville, Tennessee

${ }^{*}$ Speaker. 


\section{Background}

Jefferson Lab has approved two experiments, E12-13-011 to measure the tensor structure function $b_{1}$ [1] and E12-15-005 to measure the tensor asymmetry $A_{z z}$ [2]. These experiments will probe sea quark effects [3], exotic 6-quark states [4], short range correlations [5], and to further our understanding of how an atomic nucleus arises from it's constituent quarks and gluons. Both of these experiments are approved on the condition of demonstrating a high-luminosity and tensor-polarized target capable of achieving tensor polarization of $P_{z z}>30 \%$.

Recent advances in polarized targets have massively improved our understanding of the internal structure of nucleons by introducing a new degree of freedom, but require experiments to be run at much higher momenta where statistics are limited to probe similar physics as $A_{z z}$ and cannot access observables such as $b_{1}$ that are non-zero only with $\left|P_{z z}\right|>0$. The development of tensor polarization is required to probing short-distance high-momentum dynamics where theoretical models differ by up to a factor of two and can be determined to $6 \sigma$ in $1 / 6$ the time compared to traditional methods [2].

The University of New Hampshire (UNH) polarized target lab has recently come on-line and demonstrated vector polarization enhancement utilizing a novel solid-state microwave source,multiple nuclear magnetic resonance (NMR) devices for polarization measurement, including a Liverpool Q-meter, a Lawrence Berkeley National Lab VME Q-meter, and multiple low-cost vector network analyzers, and 3D printed target cups and ladders.

\section{System Overview}

The typical DNP target consists of a superconducting magnet to polarize electrons in the target material, a helium evaporation refrigerator to maximize electron polarization, a high-power millimeter wave to transfer electron polarization to nuclear polarization, and an NMR system to measure the amount of nuclear polarization. A review of the process is given in detail in [6], and a generalized schematic for these subsystems implemented in the University of New Hampshire (UNH) Polarized Target Lab is shown in Fig. 1. Although there is continued work being done to optimize the system, the UNH Polarized Target Lab has demonstrated proton polarization enhancement on TEPMO-doped Araldite and butanol, as shown in Fig. 2.

\section{Millimeter Wave System}

The UNH Polarized Target Lab utilizes recent advances in solid-state millimeter-wave (mmwave) systems that have been pioneered by the medical and NMR spectroscopy communities [7]. A circularly polarized $140 \pm 2 \mathrm{GHz} \mathrm{mm}$-wave system consisting of an up-to $500 \mathrm{~mW}$ solid-state source connected to ultra-low-loss $(<0.1 \mathrm{~dB} / \mathrm{m})$ overmodal waveguides via a quasi-optical bridge has been installed and demonstrated successful vector polarization enhancement on protons at UNH. A schematic of this system is shown in Fig. 3. A beam polarizer is placed in front of a polarization transforming mirror before the beam is transmitted to the waveguide, which prevents a reflected beam from re-entering the horn that could damage the system. Although the mm-wave power on target is approximately a factor of $1 / 2$ that of traditional EIO-based systems, initial studies have indicated that final polarization enhancement saturates below the maximum power of the 




Figure 1: Overview of (a) the UNH Polarized Target System with insert (b) highlighting the region around the target cup.

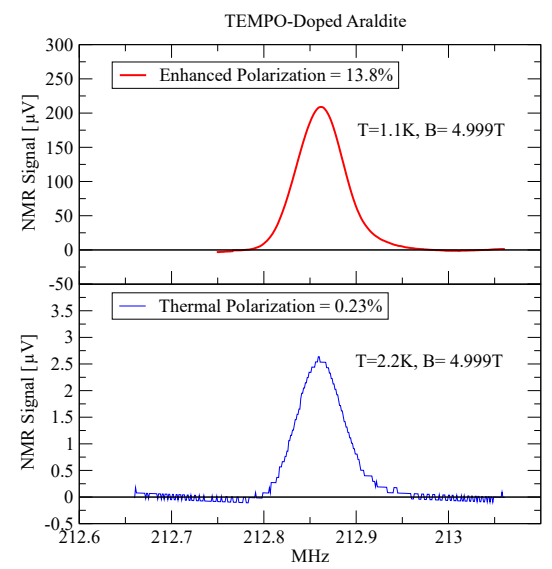

Figure 2: The UNH Polarized Target Lab has demonstrated initial DNP enhancement on TEMPO-doped materials.

system as shown in Fig. 4. Additionally, the use of a circularly polarized mm-wave has been shown by other groups to increase increase polarization enhancement on the order of $30 \%$ above that obtained with a linearly polarized mm-wave beam [8].

\section{D Printed Target Cups and Ladders}

Recent advances in additive manufacturing allow for rapid prototyping and development of arbitrarily complex geometries. The UNH Polarized Target Lab has utilized these techniques to develop target cups and ladders.

These cups are made of a Teflon-like fluoroplastic called Kel-F that both maintains roomtemperature properties down to $1 \mathrm{~K}$ and doesn't contain free protons that would introduce noise in the NMR system. 3D-printable Kel-F filament is unfortunately not currently available for purchase, so the printing filament needs to be custom made. We have developed a custom extruder to produce 


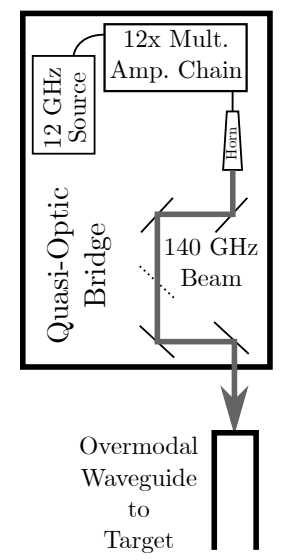

Figure 3: A circularly polarized $140 \mathrm{GHz}$ beam is created by producing a $12 \mathrm{GHz}$ signal that is sent through a 12x multiplier-amplifier chain. The output signal is then coupled to a quasi-optic bridge which transmits the beam through air into an overmodal waveguide.

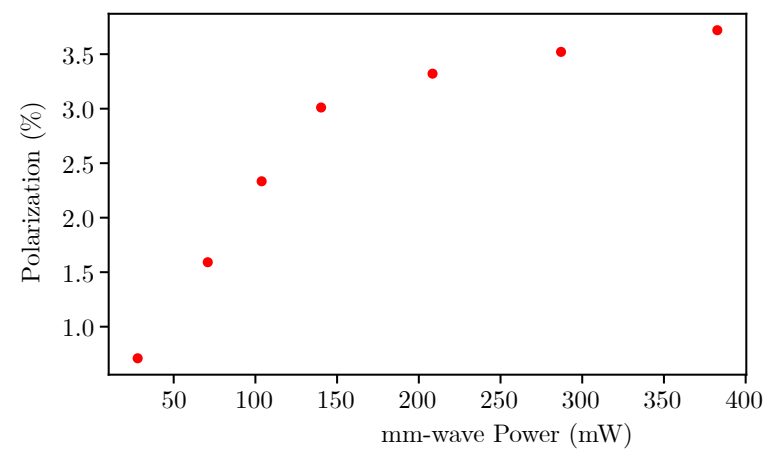

Figure 4: DNP enhancement at $4.2 \mathrm{~K}$ on Araldite indicates that the maximum polarization enhancement in the UNH system saturates when the mm-wave power reaches approximately $150 \mathrm{~mW}$.

3D-printable Kel-F filament that has been used in a commercially-available 3D printer that was modified to handle the high temperatures required to process Kel-F and to minimize decomposition of the material into hazardous gasses such as $\mathrm{HF}$ and $\mathrm{HCl}$ [9]. Utilizing this technique, we have 3D printed Kel-F target cups that have been used in recent polarization measurements, as shown in Fig. 5. Also shown is a target ladder that the Kel-F cups are mounted on to. The ladder is made of a 3D-printable material from Form Labs called 'Durable,' which survives repeated temperature cycling between $1 \mathrm{~K}$ and room temperature.

\section{Software-Defined-Radio-based NMR Measurements}

The development of software-defined-radio (SDR) has recently brought down the price of vector network analyzers (VNA) dramatically. Along with more traditional systems such as a Liverpool Q-meter and a VME crate-based NMR system developed at LANL [10], the UNH Polarized Target Lab is using SDR-based VNAs to measure NMR spectra to determine polarization. These systems offer advantages in their low cost, their ability to measure an extremely wide frequency range without requiring extra circuitry, and in their ability to simultaneously measure the real and imaginary components of the impedance, whereas a Q-meter-based system only measures the real 


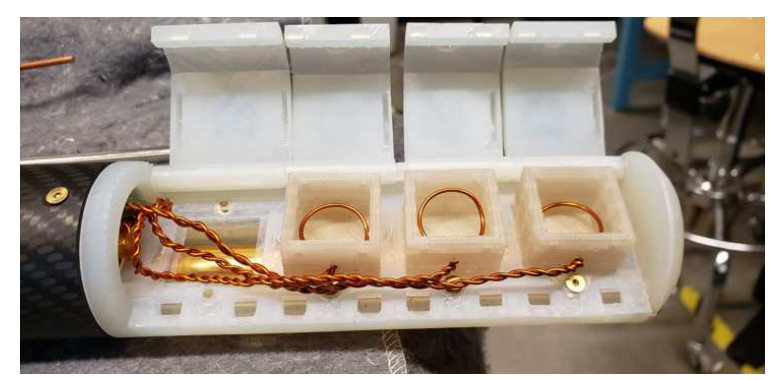

Figure 5: Cubic 3D printed Kel-F cups were mounted to a 3D printed target ladder and used in a recent cool-down.

component for NMR analysis. Plotting the NMR spectra along with a wide portion of the background Q-curve along the real and imaginary parts of the impedance creates a circular background with an NMR signal on top. This circular background is easily fit and subtracted, eliminating the need for "wing fits" that are traditionally used to remove the Q-curve background. An example of this technique utilized on an enhanced proton from TEMPO-doped Araldite is shown in Fig. 6.

\section{Summary}

The UNH Polarized Target Lab has recently demonstrated polarization enhancement via dynamic nuclear polarization on a system using a novel solid-state $140 \mathrm{GHz}$ source, 3D printed Kel-F target cups, and is utilizing a low-cost SDR-based VNA alongside traditional Q-meters to measure polarization. The UNH Nuclear Physics Group is using this new target system to develop tensor polarization greater than $30 \%$ to meet the conditions on multiple approved Jefferson Lab experiments.

\section{References}

[1] K. Slifer, E. Long, D. Keller, O. Rondon, P. Solvignon, N. Kalantarians et al., The Tensor Structure Function, $b_{1}$, JLab PAC40, C12-13-011 (2013) .

[2] E. Long, D. Keller, K. Slifer, D. Day, P. Solvignon, D. Higinbotham et al., Measurements of the Quasi-Elastic and Elastic Deuteron Tensor Asymmetries, JLab PAC44, C12-15-005 (2016) .

[3] S. Kumano, Tensor-polarized quark and antiquark distribution functions in a spin-one hadron, Phys. Rev. D82 (2010) 017501 [1005.4524].

[4] G. A. Miller, Pionic and Hidden-Color, Six-Quark Contributions to the Deuteron b1 Structure Function, Phys.Rev. C89 (2014) 045203 [1311.4561].

[5] M. Sargsian, Inclusive and Exclusive Scatterings from Tensor Polarized Deuteron, J.Phys. Conf.Ser. 543 (2014) 012009 [1409.6056].

[6] D. Crabb et al., Solid polarized targets for nuclear and particle physics experiments, Ann.Rev.Nucl.Part.Sci. 47 (1997) 67.

[7] T. A. Siaw et al., A versatile and modular quasi optics-based $200 \mathrm{ghz}$ dual dynamic nuclear polarization and electron paramagnetic resonance instrument, J.Mag.Res. 264 (2016) 131. 

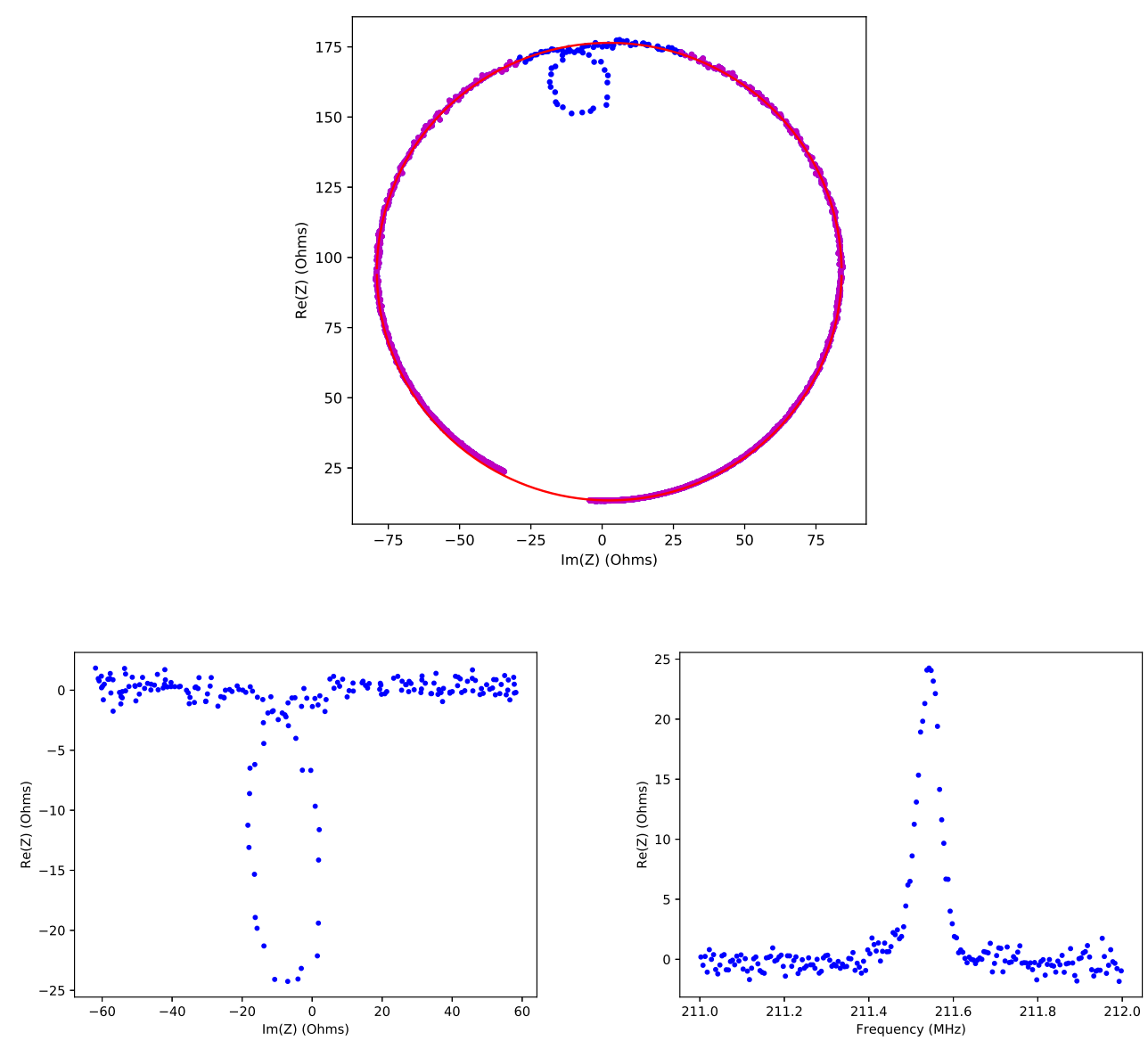

Figure 6: An enhanced proton signal was measured in terms of the real and imaginary components of impedance using an SDR-based VNA. Plotted this way, the background Q-curve is easily fit by a circle (top). The circle fit can then be removed (bottom left), and the real part of the impedance plotted against frequency (bottom right) to measure the NMR signal.

[8] B. D. Armstrong, D. T. Edwards, R. J. Wylde, S. A. Walker and S. Han, A 200 ghz dynamic nuclear polarization spectrometer, Phys. Chem. Chem. Phys. 12 (2010) 5920.

[9] K. McGuire and E. Long, Fabrication of 3D-printed PCTFE material cups for dynamic nuclear polarization target at cryogenic temperatures, 1910.13254.

[10] P. McGaughey. Private Communication. 